\title{
Centralized Pan-Middle East Survey on the Under- Treatment of Hypercholesterolemia: Results from the CEPHEUS II Study in Egypt
}

\author{
Ashraf Reda - Alaa Etman · Ali Abdel-Rahim • Nabil Farag • \\ Osama Sanad $\cdot$ Sameh Salamah
}

Received: February 23, 2017 / Published online: March 29, 2017

(C) The Author(s) 2017. This article is an open access publication

\section{ABSTRACT}

Introduction: As part of the CEPHEUS study, CEPHEUS I was conducted in 2010 and 2011 in Cairo and then the CEPHEUS II study was carried out in Alexandria and Delta Regions in Egypt between April 2014 and August 2015 to determine the proportion of dyslipidemic

Enhanced content To view enhanced content for this article, go to http://www.medengine.com/Redeem/ 9408F0603D4A3C5E.

A. Reda $(\bowtie)$

Monofya University, Egypt, 13127 Mohamed Fareed

Street, Bab El-Louk, Cairo, Egypt

e-mail: ashrafreda5555@gmail.com

A. Etman

National Heart Institute, Egypt, 1 Mohamed

Mahmoud Street, Bab El-Louk, Cairo, Egypt

A. Abdel-Rahim

Alexandria University, Egypt, 8 Kamel Morsy Street, El-Shatby, Alexandria, Egypt

N. Farag

Ain Shams University, Egypt, 71 Elhegaz Street, Heliopolis, Cairo, Egypt

O. Sanad

Benha University, Egypt, 31 Wahby Street,

El-Manshia, Benha, Egypt

S. Salamah

Cairo University, Egypt, 12 Dokki Street, Dokki,

Giza, Egypt patients on lipid-lowering treatment reaching LDL-C treatment goals.

Methods: We conducted an open-label, observational, multicenter, cross-sectional survey where 90 investigators enrolled 1127 patients receiving lipid-lowering drugs for at least 3 months. After signing informed consent forms, the study questionnaires were completed by patients and investigators. Blood samples were taken for laboratory investigations. Patients with missing LDL-C data were excluded from the analysis and results from 896 patients were analyzed according to European Atherosclerosis Society and EAS/ESC 2011 guidelines.

Results: Out of 896 patients enrolled based on the risk stratification of EAS/ESC 2011 guidelines, $12.4 \%$ were classified as low risk, $20.0 \%$ as moderate risk, $2.5 \%$ as high risk, and $65.2 \%$ as very high risk. Achievement goals were 84.7, $44.7,18.2$, and $22.3 \%$ for low-risk, moderate-risk, high-risk, and very high risk patients, respectively, with an overall achievement goal of $34.4 \%$. The study population included $50.2 \%$ diabetes, $64.4 \%$ hypertension, $54.9 \%$ metabolic syndrome, $32.2 \%$ family history of cardiovascular disease, $23.1 \%$ smokers, and $33.8 \%$ secondary prevention. Lipid-lowering agents were prescribed as a monotherapy to $90.1 \%$ and in combination in $9.9 \%$ with goal achievements of 34 and $38 \%$, respectively ( $p>0.05)$. Statins were prescribed to $86.9 \%$ of patients. The most frequent prescribed statins were rosuvastatin 
(47.1\%) and atorvastatin (36.0\%), followed by simvastatin $(9.2 \%)$. Treatment goal was achieved in $34.2,36.0$, and $31.7 \%$ for rosuvastatin, atorvastatin, and simvastatin, respectively, with no significant difference in achievement goals $(p>0.05)$.

Conclusions: Hypercholesterolemia is still not being effectively managed in many at-risk patients in Egypt. The majority of patients enrolled in the study were being actively treated with lipid-lowering medications yet the percentage goal achievement was less when compared to CEPHEUS results.

Keywords: Cardiovascular; CVD; Cardiovascular risk; CEPHEUS; Egypt; Hypercholesterolemia; Statins

\section{INTRODUCTION}

Cardiovascular diseases (CVD) are the leading cause of death worldwide. Multiple risk factors contribute to CVD. A recent standardized case-control study in 52 countries specifically focusing on acute myocardial infarction identified that six risk factors (dyslipidemia, smoking, hypertension, diabetes mellitus, abdominal obesity, and stressful psychosocial factors) account for $90 \%$ of myocardial infarctions in men and $94 \%$ in women [1].

Epidemiological surveys have shown that elevated total serum cholesterol (TC) and particular elevated low-density lipoprotein cholesterol (LDL-C) levels are strongly correlated with CHD risk. Many guidelines identify LDL-C as the primary target of cholesterol-lowering therapy and recommend LDL-C goals either numerical or percentage of reduction that are based on the risk category a patient belongs to $[2,3]$.

Hypercholesterolemia treatment has shown clear benefits in the primary and secondary prevention of CVD. However, cross-sectional surveys conducted in Europe [5, 6], and the USA [6-8] on the management of risk factors in patients with CHD have indicated that hypercholesterolemia continues to be inadequately treated [4-6]. Accordingly, many studies are required to assess the current level of under-treatment of hypercholesterolemia, defined as receiving lipid-lowering drug therapy but having uncontrolled TC and/or LDL-C levels and to understand better why patients on pharmacological treatment do not achieve their treatment goals [4-7].

This study is part of the worldwide conducted CEPHEUS studies; Centralized Pan-Middle East Survey on the under-treatment of hypercholesterolemia and the second wave of the Centralized Pan-Middle East Survey on the under-treatment of hypercholesterolemia: results from the CEPHEUS Study in Egypt [8]. The current study is based on the European Atherosclerosis Society 2011 and the European Society of Cardiology guidelines (EAS/ESC 2011) [8]. The primary objective is to determine the proportion of patients on lipid-lowering pharmacological treatment reaching the LDL-C goals in Egypt according to the EAS/ESC 2011 guidelines. Secondary objectives were to determine the proportions in pre-defined subpopulations including primary and secondary prevention, metabolic syndrome, patients' risk profile category, and identification of determinants (patient and physician characteristics) for patients not reaching their treatment goals and physician characteristics associated with the allocation of different treatment regimens.

\section{METHODS}

This is an open-label, observational, non-interventional, multicenter, single-visit cross-sectional survey conducted on patients under lipid-lowering pharmacological treatment. Ninety investigators enrolled 1127 patients on lipid-lowering drugs between April 24, 2014 and August 29, 2015 from Alexandria and Delta Regions in Egypt as part of the CEPHEUS study (NIS-EG-CRE-2012/01). Patients of age 18 years or more receiving lipid-lowering drug for at least 3 months with no dose change for at least 6 weeks signed informed consent to participate and comply with study procedures. Before assessment, after signing informed patient consent, the study questionnaires were completed by patients and investigators. The investigators completed case record forms (CRF) with 
the patient's demographics, known cardiovascular risk factors, cardiovascular medical history, current lipid-lowering drug treatment, and the reason for the current therapy. Samples were taken for total cholesterol, LDL cholesterol, HDL cholesterol, triglycerides, HbA1c, and the ratio of total cholesterol/HDL-C was calculated. The investigators received results within 5 days and completed the CRFs. Under-treatment was defined as receiving lipid-lowering pharmacological treatment and not reaching the LDL-C goals according to the EAS/ESC 2011 guidelines. Non-HDL-C goals was defined as patients with HDL-c $<40 \mathrm{mg} / \mathrm{dl}$ and fasting triglycerides $>200 \mathrm{mg} / \mathrm{dl}$.

Among the 1127 enrolled patients, patients with missed LDL-C data $(n=231)$ were excluded from the analysis and results from 896 patients are presented here.

\section{Statistical Analysis}

Data of 896 subjects were analyzed in this study. This gives a margin error of $\pm 3.2 \%$ at $95 \%$ confidence level with expected number of patients achieving treatment goals at 50\%.

Number and percentage ( $n$ and \%) presented the categorical data and Chi-square test (or its subsidiaries) was used to obtain $p$ values to test significance for difference between groups. Descriptive statistics (mean \pm SD) presented the numerical data and Student's $t$ test (or its subsidiaries) was used to obtain $p$ values to test significance for difference between groups. The calculation of statistics and proportions did not include the missing data. Missing values were presented in separate tables.

\section{Compliance with Ethics Guidelines}

All procedures followed were in accordance with the ethical standards of the responsible committee on human experimentation (institutional and national) and with the Helsinki Declaration of 1964, as revised in 2013. Informed consent was obtained from all patients for being included in the study. This article does not contain any new studies with human or animal subjects performed by any of the authors.

\section{RESULTS}

Eight hundred ninety-six subjects were included in the analysis. The mean age was $53.9 \pm 10.7$ years and $57.6 \%$ were male. The main reason of pharmacological therapy was for primary prevention with $59.4 \%$ of patients. Statins were the most prescribed agents with $95.2 \%$ (853 of 896 ) of patients; $90 \%$ (768) used statins as monotherapy and 10\% (85) in combined therapy. Rosuvastatin was the most frequently prescribed statin. It was prescribed to $47.1 \%$ (422 of 896 ) from which 391 were monotherapy and 31 were combined therapy. Other and more detailed demographic data and patients characteristics are shown in Table 1.

\section{LDL Target Goal Achievement}

Overall, 34.4\% (308 of 896 patients) achieved their LDL-C target goal according to the EAS/ ESC 2011 guidelines.

A total of $84.7 \%$ of low-risk patients achieved target LDL-C goal $(<155 \mathrm{mg} / \mathrm{dl}), 44.7 \%$ of moderate-risk patients achieved target LDL-C goal $(<115 \mathrm{mg} / \mathrm{dl}), 18.2 \%$ of high-risk patients achieved target LDL-C goal $(<100 \mathrm{mg} / \mathrm{dl})$ and $22.3 \%$ of very high risk patients achieved target LDL-C goal ( $<70 \mathrm{mg} / \mathrm{dl}$ or $\geq 50 \%$ reduction).

From the patients who were on rosuvastatin, $33.2 \%$ reached the goal with monotherapy and $48.4 \%$ with combined therapy, with an overall achievement of $34.2 \%$. From patients who were prescribed treatment for primary prevention: $36.1 \%$ achieved LDL-C goal, for secondary prevention $29.9 \%$, and familial hypercholesterolemia $36.2 \%$. $\quad(p=0.082)$. For special populations, the goal was reached for $67.7 \%$ of patients with metabolic syndrome and $63.1 \%$ without ( $p=0.087)$; the goal was reached for $21.6 \%$ of patients with diabetes mellitus and $46.5 \%$ without $(p<0.001)$, and finally, goal was reached for $29.6 \%$ of patients with arterial hypertension and $42.6 \%$ without $(p<0.001)$ (Tables 2, 3). 
Table 1 Patients' demography and other variables at the baseline visit

\begin{tabular}{|c|c|c|c|c|c|}
\hline \multirow{2}{*}{$\begin{array}{l}\text { Parameter } \\
\text { Age }\end{array}$} & \multirow[t]{2}{*}{ Variable } & \multirow{2}{*}{$\begin{array}{l}\text { Valid answer } \\
\text { (base) }\end{array}$} & \multirow{2}{*}{$\begin{array}{c}\text { Missing } \\
0\end{array}$} & \multicolumn{2}{|c|}{$\begin{array}{l}\text { Mean and SD } \\
\text { or } N \text { and } \%\end{array}$} \\
\hline & & & & 53.9 & 10.7 \\
\hline Weight & & 892 & 4 & 92.3 & 17.1 \\
\hline Height & & 887 & 9 & 168.8 & 8.8 \\
\hline BMI & & 887 & 9 & 32.5 & 6.4 \\
\hline Waist circumference & & 857 & 39 & 99.5 & 21.3 \\
\hline Systolic BP & & 893 & 3 & 133.9 & 15.9 \\
\hline Diastolic BP & & 893 & 3 & 84.4 & 9.1 \\
\hline \multirow[t]{2}{*}{ Gender } & Male, $n(\%)$ & 896 & 0 & 516 & $57.6 \%$ \\
\hline & Female, $n(\%)$ & & & 380 & $42.4 \%$ \\
\hline \multirow[t]{2}{*}{ Nationality } & Egyptian, $n(\%)$ & 896 & 31 & 859 & $99.3 \%$ \\
\hline & Other (Syrian), $n(\%)$ & & & 6 & $0.7 \%$ \\
\hline \multirow[t]{3}{*}{ Ethnicity } & $\begin{array}{l}\text { Middle Eastern (including North } \\
\text { Africa) }\end{array}$ & 869 & 27 & 857 & $98.6 \%$ \\
\hline & $\begin{array}{l}\text { Caucasian (including Europe and } \\
\text { North America) }\end{array}$ & & & 6 & $0.7 \%$ \\
\hline & Other, $n(\%)$ & & & 6 & $0.7 \%$ \\
\hline \multirow{3}{*}{$\begin{array}{l}\text { Reason for } \\
\text { pharmacological therapy }\end{array}$} & Primary prevention & 876 & 20 & 520 & $59.4 \%$ \\
\hline & Secondary prevention & & & 296 & $33.8 \%$ \\
\hline & Familial hypercholesterolemia & & & 60 & $6.8 \%$ \\
\hline \multirow[t]{4}{*}{ Medical history } & $\begin{array}{l}\text { History of coronary heart disease, } \\
n(\%)\end{array}$ & 896 & 0 & 309 & $34.5 \%$ \\
\hline & $\begin{array}{l}\text { History of peripheral artery disease, } \\
n(\%)\end{array}$ & 894 & 2 & 118 & $13.2 \%$ \\
\hline & $\begin{array}{l}\text { Cerebrovascular atherosclerotic } \\
\text { disease, } n(\%)\end{array}$ & 896 & 0 & 62 & $6.9 \%$ \\
\hline & Established CVD & 886 & 10 & 376 & $42.4 \%$ \\
\hline \multirow[t]{4}{*}{ Comorbid condition } & Arterial hypertension, $n$ (\%) & 871 & 25 & 561 & $64.4 \%$ \\
\hline & Diabetes, $n(\%)$ & 877 & 19 & 440 & $50.2 \%$ \\
\hline & $\begin{array}{l}\text { Family history of premature } \\
\text { cardiovascular disease, } n(\%)\end{array}$ & 845 & 51 & 272 & $32.2 \%$ \\
\hline & Current smoker, $n(\%)$ & 872 & 10 & 207 & $23.1 \%$ \\
\hline
\end{tabular}


Table 1 continued

\begin{tabular}{|c|c|c|c|c|c|}
\hline \multirow{2}{*}{$\begin{array}{l}\text { Parameter } \\
\text { Lab test }\end{array}$} & \multirow{2}{*}{$\begin{array}{l}\text { Variable } \\
\text { Total cholesterol, } \mathrm{mg} / \mathrm{dl}(\mathrm{mmol} / \mathrm{l})\end{array}$} & \multirow{2}{*}{$\begin{array}{l}\begin{array}{l}\text { Valid answer } \\
\text { (base) }\end{array} \\
896\end{array}$} & \multirow{2}{*}{$\begin{array}{c}\text { Missing } \\
0\end{array}$} & \multicolumn{2}{|c|}{$\begin{array}{l}\text { Mean and SD } \\
\text { or } N \text { and } \%\end{array}$} \\
\hline & & & & 189.3 & 47.6 \\
\hline & HDL-C, mg/dl (mmol/l) & 896 & 0 & 45.2 & 13 \\
\hline & LDL-C, mg/dl (mmol/l) & 896 & 0 & 115 & 41.7 \\
\hline & Triglycerides, mg/dl (mmol/l) & 894 & 2 & 150.9 & 84 \\
\hline & Total cholesterol/HDL-C ratio & 2 & 894 & 5 & 1.4 \\
\hline & Glucose, mg/l & 716 & 180 & 131.9 & 61.1 \\
\hline & $\mathrm{HbAlc}, \%(\mathrm{mmol} / \mathrm{mol})$ & 891 & 5 & 7.1 & 1.9 \\
\hline \multirow{6}{*}{$\begin{array}{l}\text { Current prescribed } \\
\text { medication groups }\end{array}$} & Statin monotherapy & 885 & 11 & 768 & $85.7 \%$ \\
\hline & Statin + fibrate & & & 47 & $5.2 \%$ \\
\hline & Statin + ezetimibe & & & 35 & $3.9 \%$ \\
\hline & Ezetimibe monotherapy & & & 25 & $2.8 \%$ \\
\hline & Fibrate monotherapy & & & 7 & $0.8 \%$ \\
\hline & Statin + fibrate + ezetimibe & & & 3 & $0.3 \%$ \\
\hline \multirow{11}{*}{$\begin{array}{l}\text { Current prescribed } \\
\text { monotherapy drugs }\end{array}$} & Rosuvastatin & 885 & 11 & 422 & $47.7 \%$ \\
\hline & Atorvastatin & & & 323 & $36.5 \%$ \\
\hline & Simvastatin & & & 82 & $9.3 \%$ \\
\hline & Ezetimibe & & & 63 & $7.1 \%$ \\
\hline & Fenofibrate & & & 56 & $6.3 \%$ \\
\hline & Fluvastatin & & & 10 & $1.1 \%$ \\
\hline & Lovastatin & & & 3 & $0.3 \%$ \\
\hline & Pravastatin & & & 3 & $0.3 \%$ \\
\hline & Bezafibrate & & & 1 & $0.1 \%$ \\
\hline & Gemfibrozil & & & 1 & $0.1 \%$ \\
\hline & Undefined statins & & & 32 & $3.6 \%$ \\
\hline
\end{tabular}

\section{Results of the Investigators' Questionnaire}

There were $41.4 \%$ of the investigators that mentioned that they use EAS/ESC 2011 guidelines. Most of the investigators use guidelines to set individual cholesterol targets; $94.4 \%$ depend on LDL-C as an indicator.
Percent split of prescriptions as reported by investigators was $86.9 \%$ for statins, $9.5 \%$ for fibrates, and $3.6 \%$ for bile acid sequestrants and other drugs. Investigators review their patients mostly every 3 months, and they stated that an average of $55.6 \%$ of patients achieved their target cholesterol level. Detailed results of the investigators' questionnaire are presented in Table 4. 
Table 2 EAS/ESC 2011 risk-profile category, number and percent in each group, lipid-lowering drug, mean and SD of the used dose and number and percent of patients achieving the LD-C treatment goal

\begin{tabular}{|c|c|c|c|c|c|c|c|c|}
\hline \multirow{3}{*}{$\begin{array}{l}\text { EAS/ESC } 2011 \text { risk } \\
\text { profile category }\end{array}$} & \multirow{2}{*}{\multicolumn{2}{|c|}{ Base }} & \multicolumn{6}{|c|}{ Lipid-lowering agents } \\
\hline & & & \multicolumn{2}{|l|}{ Agent } & \multicolumn{2}{|l|}{ Dose } & \multicolumn{2}{|c|}{ Achieved treatment goal } \\
\hline & Base & $\%$ & Name & Base & $\overline{\text { Mean }}$ & SD & $\bar{N}$ & $\%$ \\
\hline \multirow[t]{11}{*}{ All } & 896 & & Rosuvastatin & 442 & 14.6 & 5.1 & 152 & 34.4 \\
\hline & & & Atorvastatin & 323 & 24.8 & 14.8 & 118 & 36.5 \\
\hline & & & Simvastatin & 82 & 25.7 & 16.2 & 26 & 31.7 \\
\hline & & & Ezetimibe & 63 & 15.0 & 7.1 & 26 & 41.3 \\
\hline & & & Fenofibrate & 56 & 260.0 & 64.8 & 21 & 37.5 \\
\hline & & & Fluvastatin & 10 & 10.0 & & 1 & 10.0 \\
\hline & & & Pravastatin & 3 & 20.0 & & 1 & 33.3 \\
\hline & & & Lovastatin & 3 & 40.0 & & 1 & 33.3 \\
\hline & & & Bezafibrate & 1 & & & 0 & 0.0 \\
\hline & & & Gemfibrozil & 1 & & & 0 & 0.0 \\
\hline & & & Undefined statin & 32 & 21.1 & 11.7 & 9 & 28.1 \\
\hline \multirow[t]{11}{*}{ Very high risk } & 584 & $65.2 \%$ & Rosuvastatin & 272 & 16.3 & 5.2 & 56 & 20.6 \\
\hline & & & Atorvastatin & 215 & 25.1 & 14.2 & 57 & 26.5 \\
\hline & & & Simvastatin & 52 & 31.0 & 20.2 & 10 & 19.2 \\
\hline & & & Ezetimibe & 40 & 14.2 & 5.1 & 12 & 30.0 \\
\hline & & & Fenofibrate & 33 & 253.3 & 70.0 & 9 & 27.3 \\
\hline & & & Fluvastatin & 8 & & & 0 & 0.0 \\
\hline & & & Pravastatin & 2 & 20.0 & & 1 & 50.0 \\
\hline & & & Lovastatin & 1 & & & 0 & 0.0 \\
\hline & & & Bezafibrate & 1 & & & 0 & 0.0 \\
\hline & & & Gemfibrozil & 1 & & & 0 & 0.0 \\
\hline & & & Undefined statin & 22 & 26.7 & 11.5 & 3 & 13.6 \\
\hline \multirow[t]{5}{*}{ High risk } & 22 & $2.5 \%$ & Rosuvastatin & 11 & 15.0 & 7.1 & 2 & 18.2 \\
\hline & & & Atorvastatin & 7 & 15.0 & 7.1 & 2 & 28.6 \\
\hline & & & Fenofibrate & 3 & 300.0 & & 1 & 33.3 \\
\hline & & & Simvastatin & 2 & & & 0 & 0.0 \\
\hline & & & Ezetimibe & 2 & & & 0 & 0.0 \\
\hline
\end{tabular}


Table 2 continued

\begin{tabular}{|c|c|c|c|c|c|c|c|c|}
\hline \multirow{3}{*}{$\begin{array}{l}\text { EAS/ESC } 2011 \text { risk } \\
\text { profile category }\end{array}$} & \multirow{2}{*}{\multicolumn{2}{|c|}{ Base }} & \multicolumn{6}{|c|}{ Lipid-lowering agents } \\
\hline & & & \multicolumn{2}{|l|}{ Agent } & \multicolumn{2}{|l|}{ Dose } & \multicolumn{2}{|c|}{ Achieved treatment goal } \\
\hline & Base & $\%$ & Name & Base & Mean & SD & $N$ & $\%$ \\
\hline \multirow[t]{9}{*}{ Moderate risk } & 179 & $20.0 \%$ & Rosuvastatin & 88 & 13.4 & 4.8 & 47 & 53.4 \\
\hline & & & Atorvastatin & 61 & 23.5 & 11.9 & 23 & 37.7 \\
\hline & & & Simvastatin & 16 & 23.3 & 15.3 & 3 & 18.8 \\
\hline & & & Ezetimibe & 15 & 17.5 & 10.4 & 8 & 53.3 \\
\hline & & & Fenofibrate & 13 & 272.0 & 62.6 & 5 & 38.5 \\
\hline & & & Fluvastatin & 1 & & & 0 & 0.0 \\
\hline & & & Lovastatin & 1 & & & 0 & 0.0 \\
\hline & & & Pravastatin & 1 & & & 0 & 0.0 \\
\hline & & & Undefined statin & 5 & 10.0 & & 1 & 20.0 \\
\hline \multirow[t]{8}{*}{ Low risk } & 111 & $12.4 \%$ & Rosuvastatin & 51 & 13.8 & 4.9 & 40 & 78.4 \\
\hline & & & Atorvastatin & 40 & 25.8 & 17.8 & 36 & 90.0 \\
\hline & & & Simvastatin & 12 & 21.0 & 11.0 & 10 & 83.3 \\
\hline & & & Fenofibrate & 7 & 253.3 & 72.3 & 6 & 85.7 \\
\hline & & & Ezetimibe & 6 & 13.3 & 5.2 & 6 & 100.0 \\
\hline & & & Fluvastatin & 1 & 10.0 & & 1 & 100.0 \\
\hline & & & Lovastatin & 1 & 40.0 & & 1 & 100.0 \\
\hline & & & Undefined statin & 5 & 20.0 & 12.2 & 5 & 100.0 \\
\hline
\end{tabular}

Eighty-five patients were on combined-drug therapy. Each patient was on an average of 1.1 drugs

\section{Results of the Patients' Questionnaire}

Eight hundred ninety-two patients returned completed questionnaires. More than $75 \%$ of the patients were aware of good and bad cholesterol and mentioned that their doctor told them about their level of cholesterol and target goals and discussed the proper lifestyle, diet, and prescribed medications; $78.5 \%$ mentioned that they were satisfied with the information they had about high cholesterol. Results showed that patients were trying to take drugs as they were prescribed but $41 \%$ mentioned that they sometimes forgot to take the tablet every day. Most of the patients reported that they did not change the drug they take and few of them (3.3\%) reported that they had changed the prescribed drug more than two times. Of the patients, 49\% stated that they achieved their target cholesterol level and $75 \%$ were satisfied with the treatment they were taking. A strong correlation was found between achieving treatment goal as obtained from CEPHEUS II results and patient compliance as obtained from patients' questionnaire $(p<0.001)$. Table 5 shows detailed results of the patients' questionnaire.

\section{DISCUSSION}

CEPHEUS surveys in different countries worldwide have aimed to investigate the proportion of patients receiving lipid-lowering drugs and 
Table 3 LDL-C target goal achievement according to EAS/ESC 2011 guidelines by different sample determinants

\begin{tabular}{|c|c|c|c|c|c|c|c|}
\hline \multirow[t]{2}{*}{ Determinant } & \multirow[t]{2}{*}{ Variable } & \multirow[t]{2}{*}{ Base } & \multicolumn{2}{|c|}{ Achieved } & \multicolumn{2}{|c|}{ Not achieved } & \multirow[t]{2}{*}{$p$ value } \\
\hline & & & $\overline{\text { Yes }}$ & $\%$ & No & $\%$ & \\
\hline \multirow[t]{3}{*}{ Reason for treatment } & Primary prevention & 520 & 189 & 36.3 & 331 & 63.7 & 0.082 \\
\hline & Secondary prevention & 296 & 86 & 29.1 & 210 & 70.9 & \\
\hline & Familial hypercholesterolemia & 60 & 23 & 38.3 & 37 & 61.7 & \\
\hline \multirow[t]{2}{*}{ Metabolic syndrome } & Metabolic syndrome & 492 & 159 & 32.3 & 333 & 67.7 & 0.087 \\
\hline & Non-metabolic syndrome & 404 & 149 & 36.9 & 255 & 63.1 & \\
\hline \multirow[t]{2}{*}{ Gender } & Male & 516 & 167 & 32.4 & 349 & 67.6 & 0.140 \\
\hline & Female & 380 & 141 & 37.1 & 239 & 62.9 & \\
\hline \multirow[t]{12}{*}{ Age distribution } & $<20$ years & 1 & 0 & 0.0 & 1 & 100.0 & 0.081 \\
\hline & 20-34 years & 40 & 21 & 52.5 & 19 & 47.5 & \\
\hline & $35-39$ years & 39 & 19 & 48.7 & 20 & 51.3 & \\
\hline & $40-44$ years & 89 & 34 & 38.2 & 55 & 61.8 & \\
\hline & $45-49$ years & 132 & 45 & 34.1 & 87 & 65.9 & \\
\hline & $50-54$ years & 170 & 60 & 35.3 & 110 & 64.7 & \\
\hline & $55-59$ years & 163 & 50 & 30.7 & 113 & 69.3 & \\
\hline & $60-64$ years & 122 & 32 & 26.2 & 90 & 73.8 & \\
\hline & $65-69$ years & 85 & 26 & 30.6 & 59 & 69.4 & \\
\hline & $70-74$ years & 33 & 15 & 45.5 & 18 & 54.5 & \\
\hline & $75-79$ years & 10 & 3 & 30.0 & 7 & 70.0 & \\
\hline & $>79$ years & 12 & 3 & 25.0 & 9 & 75.0 & \\
\hline \multirow[t]{3}{*}{ Ethnic origin } & $\begin{array}{l}\text { Middle Eastern } \\
\text { (including North Africa) }\end{array}$ & 857 & 297 & 34.7 & 560 & 65.3 & 0.135 \\
\hline & $\begin{array}{l}\text { Caucasian (including Europe } \\
\text { and North America) }\end{array}$ & 6 & 1 & 16.7 & 5 & 83.3 & \\
\hline & Others & 6 & 0 & 0.0 & 6 & 100.0 & \\
\hline \multirow[t]{2}{*}{ Current smoking } & Smokers & 207 & 71 & 34.3 & 136 & 65.7 & 0.971 \\
\hline & Non-smokers & 665 & 229 & 34.4 & 436 & 65.6 & \\
\hline \multirow[t]{2}{*}{ Diabetes mellitus } & Diabetics & 440 & 95 & 21.6 & 345 & 78.4 & 0.000 \\
\hline & Non-diabetics & 437 & 203 & 46.5 & 234 & 53.5 & \\
\hline \multirow[t]{2}{*}{ Arterial hypertension } & Arterial hypertension & 561 & 166 & 29.6 & 395 & 70.4 & 0.000 \\
\hline & Non-arterial hypertension & 310 & 132 & 42.6 & 178 & 57.4 & \\
\hline \multirow[t]{2}{*}{ Family history of CVD } & History of CVD & 272 & 91 & 33.5 & 181 & 66.5 & 0.643 \\
\hline & No history of CVD & 573 & 201 & 35.1 & 372 & 64.9 & \\
\hline
\end{tabular}


Table 4 Answers of investigators' questionnaire

\begin{tabular}{|c|c|c|c|c|c|}
\hline Question & Answers & $\begin{array}{l}\text { Valid } \\
\text { Answer } \\
\text { (base) }\end{array}$ & Missing & \multicolumn{2}{|c|}{$\begin{array}{l}\text { Mean \& SD } \\
\text { or } \mathbf{N} \& \%\end{array}$} \\
\hline \multicolumn{6}{|c|}{ Q1 For what proportion of patients do you set individual target cholesterol levels expressed as an actual number. } \\
\hline & & 82 & 8 & 76.9 & 27.2 \\
\hline \multicolumn{6}{|c|}{ Q2 Which lab measures do you generally use to set individual target cholesterol levels. (Yes) } \\
\hline & Q2 Total Cholesterol & 90 & 0 & 70 & $77.8 \%$ \\
\hline & Q2 HDL-C & 90 & 0 & 64 & $71.1 \%$ \\
\hline & Q2 LDL-C & 90 & 0 & 85 & $94.4 \%$ \\
\hline & Q2 Triglycerides & 90 & 0 & 67 & $74.4 \%$ \\
\hline \multicolumn{6}{|c|}{ Q3 Do you utilize any guidelines to help establish individual cholesterol targets for your patients? (yes) } \\
\hline & & 88 & 2 & 84 & $95.50 \%$ \\
\hline \multicolumn{6}{|c|}{ Q4a) Which guidelines do you use? You can give more than one answer } \\
\hline & * Joint European guidelines (SCORE) & 29 & 61 & 12 & $41.4 \%$ \\
\hline & * NCEP ATP III guidelines (FRAMINGHAM) & 29 & 61 & 13 & $44.8 \%$ \\
\hline & * National guidelines (if present) & 29 & 61 & 7 & $24.1 \%$ \\
\hline & $\begin{array}{l}\text { * Local healthcare authority } \\
\text { guidelines/recommendations }\end{array}$ & 29 & 61 & 3 & $10.3 \%$ \\
\hline & * Individual practice guidelines/recommendations & 29 & 61 & 9 & $31.0 \%$ \\
\hline & * Other (write in) & 29 & 61 & 0 & $0.0 \%$ \\
\hline & * Unable to name the precise guidelines used & 29 & 61 & 0 & $0.0 \%$ \\
\hline \multicolumn{6}{|c|}{ Q4b) Which one do you mainly use? Give only one answer } \\
\hline & * Joint European guidelines (SCORE) & 82 & 8 & 44 & $53.7 \%$ \\
\hline & * NCEP ATP III guidelines (FRAMINGHAM) & 82 & 8 & 25 & $30.5 \%$ \\
\hline & * National guidelines (if present) & 82 & 8 & 3 & $3.7 \%$ \\
\hline & $\begin{array}{l}\text { * Local healthcare authority } \\
\text { guidelines/recommendations }\end{array}$ & 82 & 8 & 0 & $0.0 \%$ \\
\hline & * Individual practice guidelines/recommendations & 82 & 8 & 6 & $7.3 \%$ \\
\hline & * Other (write in) & 82 & 8 & 4 & $4.9 \%$ \\
\hline & * Unable to name the precise guidelines used & 82 & 8 & 0 & $0.0 \%$ \\
\hline \multicolumn{6}{|c|}{$\begin{array}{l}\text { Q5 When patients are first diagnosed with hypercholesterolemia do you generally inform them of their cholesterol } \\
\text { level? }\end{array}$} \\
\hline Yes & & Yes & & 83 & $98.8 \%$ \\
\hline No & & No & & 1 & $1.2 \%$ \\
\hline \multicolumn{6}{|c|}{ Q6 In what proportion of patients do you } \\
\hline & Q6a) not mention extent of reduction at all & 86 & 4 & 8.8 & 12.2 \\
\hline & $\begin{array}{l}\text { Q6b) provide a target cholesterol level expressed as an } \\
\text { actual number }\end{array}$ & 86 & 4 & 63.1 & 28.8 \\
\hline & Q6c) provide a percentage or proportion reduction & 86 & 4 & 14.8 & 15.3 \\
\hline & $\begin{array}{l}\text { Q6d) provide a more general description such as a } \\
\text { need to reduce by 'a little' or 'a lot' }\end{array}$ & 86 & 4 & 13.3 & 15.4 \\
\hline \multicolumn{6}{|c|}{$\begin{array}{l}\text { Q7 When informing your patients which of the following types of lipid parameters measurement do you generally } \\
\text { use? You can give more than one answer (Yes) }\end{array}$} \\
\hline & Q7 Total cholesterol & 88 & 2 & 70 & $79.5 \%$ \\
\hline & Q7 HDL-C & 88 & 2 & 56 & $63.6 \%$ \\
\hline
\end{tabular}


Table 4 continued

\begin{tabular}{|c|c|c|c|c|}
\hline Q7 LDL-C & 88 & 2 & 78 & $88.6 \%$ \\
\hline Q7 Triglycerides & 88 & 2 & 59 & $67.00 \%$ \\
\hline \multicolumn{5}{|c|}{$\begin{array}{l}\text { Q8 Focusing now on pharmacological treatment for hypercholesterolemia, for what percentage of your patients do } \\
\text { you recommend treatment with: }\end{array}$} \\
\hline Q8a) Statins & 88 & 2 & 86.85 & 12.6 \\
\hline Q8b) Fibrates & 88 & 2 & 9.45 & 10.4 \\
\hline Q8c) Bile acid sequestrants & 88 & 2 & 1.42 & 3.0 \\
\hline Q8d) Other & 88 & 2 & 2.27 & 4.8 \\
\hline \multicolumn{5}{|l|}{ Q9 How frequent do you see the patient to review their cholesterol level? } \\
\hline Less frequent than once per year & 88 & 2 & 1 & $1.1 \%$ \\
\hline Once per year & 88 & 2 & 3 & $3.4 \%$ \\
\hline Once every 6 months & 88 & 2 & 12 & $13.6 \%$ \\
\hline Once every 3 months & 88 & 2 & 58 & $65.9 \%$ \\
\hline More frequent than once per 3 months & 88 & 2 & 14 & $15.9 \%$ \\
\hline \multicolumn{5}{|c|}{$\begin{array}{l}\text { Q21 In summary, thinking of all your hypercholesterolemia patients what percentages of those that have been set } \\
\text { a target cholesterol level fall into the following categories? }\end{array}$} \\
\hline $\begin{array}{l}\text { Q21a) Reached their target cholesterol level and } \\
\text { continue to stay at this level }\end{array}$ & 85 & 5 & $55.6 \%$ & $21.1 \%$ \\
\hline $\begin{array}{l}\text { Q21b) Generally stay at their target cholesterol level } \\
\text { but their cholesterol is sometimes too high }\end{array}$ & 85 & 5 & $18.8 \%$ & $11.5 \%$ \\
\hline $\begin{array}{l}\text { Q21c) Reached their target cholesterol level in the past } \\
\text { but have now lapsed }\end{array}$ & 85 & 5 & $14.7 \%$ & $10.0 \%$ \\
\hline Q21d) Have never reached their target cholesterol level & 84 & 6 & $10.8 \%$ & $12.1 \%$ \\
\hline
\end{tabular}

Q22 In general, once a patient has reached their target cholesterol level after what length of time do you ask that patient to come back to review their hypercholesterolemia? (months)

One month
2 months
3 months
6 months
12 months

Q23 What percentage of patients actually attends this review?

\begin{tabular}{|l|ll|ll} 
& 85 & 5 & $69.7 \%$ & $19.9 \%$
\end{tabular}

Q10-20 Please circle the most applicable number that meets how much you agree/disagree with each one (1 is disagree strongly and 5 is agree strongly).

The same scale will be used for all statements.

\begin{tabular}{|c|c|c|c|c|c|c|c|}
\hline Statements & Base & $\begin{array}{c}\text { Strongl } \\
y \\
\text { Disagre } \\
e \\
(1) \\
\end{array}$ & $\begin{array}{c}\text { Disagre } \\
e \\
(2) \\
\end{array}$ & $\begin{array}{l}\text { Neither } \\
\text { / } \\
\text { Nor } \\
\text { (3) }\end{array}$ & $\begin{array}{c}\text { Agree } \\
(4)\end{array}$ & $\begin{array}{l}\text { Strongl } \\
\text { y Agree } \\
(5)\end{array}$ & $\begin{array}{l}\text { Mean } \\
\text { Score }\end{array}$ \\
\hline $\begin{array}{l}\text { Q10 I feel frustrated that I am } \\
\text { not always able to effectively } \\
\text { treat my patients with } \\
\text { cardiovascular disorders }\end{array}$ & 86 & $40.7 \%$ & $16.3 \%$ & $14.0 \%$ & $11.6 \%$ & $17.4 \%$ & 2.49 \\
\hline $\begin{array}{l}\text { Q11 I find it stressful trying to } \\
\text { get my patients to their } \\
\text { cholesterol targets }\end{array}$ & 84 & $27.4 \%$ & $14.3 \%$ & $21.4 \%$ & $16.7 \%$ & $20.2 \%$ & 2.88 \\
\hline $\begin{array}{l}\text { Q12 I feel pressured to get } \\
\text { patients to their target } \\
\text { cholesterol levels }\end{array}$ & 85 & $22.4 \%$ & $14.1 \%$ & $14.1 \%$ & $20.0 \%$ & $29.4 \%$ & 3.20 \\
\hline
\end{tabular}


Table 4 continued

\begin{tabular}{|c|c|c|c|c|c|c|c|}
\hline $\begin{array}{l}\text { Q13 A sufficient number of } \\
\text { patients reach their target } \\
\text { cholesterol levels }\end{array}$ & 86 & $5.8 \%$ & $4.7 \%$ & $20.9 \%$ & $31.4 \%$ & $37.2 \%$ & 3.90 \\
\hline $\begin{array}{l}\text { Q14 I'm frustrated that the } \\
\text { guidelines instruct me to } \\
\text { advise lifestyle changes alone } \\
\text { as first line therapy in all } \\
\text { patients }\end{array}$ & 86 & $38.4 \%$ & $10.5 \%$ & $23.3 \%$ & $12.8 \%$ & $15.0 \%$ & 2.56 \\
\hline $\begin{array}{l}\text { Q15 I'm frustrated that the } \\
\text { guidelines instruct me to } \\
\text { prescribe a low dose of lipid- } \\
\text { lowering drug to all patients } \\
\text { and titrate upwards }\end{array}$ & 86 & $37.2 \%$ & $10.5 \%$ & $20.9 \%$ & $16.3 \%$ & $15.1 \%$ & 2.62 \\
\hline $\begin{array}{l}\text { Q16 I tend to prescribe a lipid } \\
\text { lowering drug only to patients } \\
\text { who have proved they can } \\
\text { adhere to diet and exercise } \\
\text { change }\end{array}$ & 85 & $49.4 \%$ & $22.4 \%$ & $10.6 \%$ & $5.8 \%$ & $11.8 \%$ & 2.08 \\
\hline $\begin{array}{l}\text { Q17 Patient compliance } \\
\text { decreases if lipid lowering } \\
\text { drugs take too long to have an } \\
\text { effect }\end{array}$ & 86 & $12.8 \%$ & $10.5 \%$ & $17.4 \%$ & $34.9 \%$ & $24.4 \%$ & 3.48 \\
\hline $\begin{array}{l}\text { Q18 I feel constrained to use } \\
\text { less effective lipid lowering } \\
\text { drugs first line }\end{array}$ & 86 & $41.9 \%$ & $20.9 \%$ & $12.8 \%$ & $10.4 \%$ & $14.0 \%$ & 2.34 \\
\hline $\begin{array}{l}\text { Q19 Patients become } \\
\text { concerned that their condition } \\
\text { is more severe if their lipid } \\
\text { lowering drug is titrated up }\end{array}$ & 86 & $12.8 \%$ & $17.5 \%$ & $26.7 \%$ & $22.1 \%$ & $20.9 \%$ & 3.21 \\
\hline $\begin{array}{l}\text { Q20 Patients become } \\
\text { concerned that their condition } \\
\text { is more severe if their lipid } \\
\text { lowering drug is frequently } \\
\text { changed }\end{array}$ & 86 & $7.0 \%$ & $15.1 \%$ & $20.9 \%$ & $33.7 \%$ & $23.3 \%$ & 3.51 \\
\hline
\end{tabular}

reaching their LDL-C treatment goals according to international guidelines. Compared to the CEPHEUS I survey that had been conducted in Egypt between October 2010 and June 2011, achievement of treatment goal was 32.5\% (339 patients out of 1043) according to the NCEP ATP III Updated 2004 guidelines [8]. CEPHEUS II in Egypt showed a slight improvement in terms of achieving treatment goals compared to CEPHEUS I according to NCEP ATP III Updated 2004 guidelines, as $39.6 \%$ (355 out of 896 ) in CEPHEUS II achieved their LDL-C treatment goal.

CEPHEUS surveys in other countries were reported and the achievement was $57 \%$ in combined Western European countries [9], 49\% for combined Asian countries [10], and 49.1\% in combined eight Asian countries (Korea, Taiwan, Thailand, Indonesia, Philippines,
Malaysia, Vietnam, Hong Kong SAR, and China) [10]. The individual results were $52 \%$ for South Africa [11], 49\% for Greece [7], 83\% for Hong Kong [12], 50\% for Taiwan [13], 31.3\% for Indonesia [14], and 53\% for Thailand [15].

Our study (CEPHEUS II) showed that the lowest target LDL-C goal achievements were obtained with diabetes mellitus $(21.6 \%)$, followed by secondary prevention (29.1\%), arterial hypertension $(29.6 \%)$, and metabolic syndrome (32.3\%). On the contrary to previous findings from the CEPHEUS Pan-Asian survey, most of our patients with diabetes mellitus and hypertension did not achieve LDL goals. It is worth mentioning that ezetimibe achieved LDL-C goal with the highest percentage of $41.3 \%$ (63 out of 89 patients), in contrast to current knowledge in the literature about statins. 
Table 5 Answers of patients' questionnaire

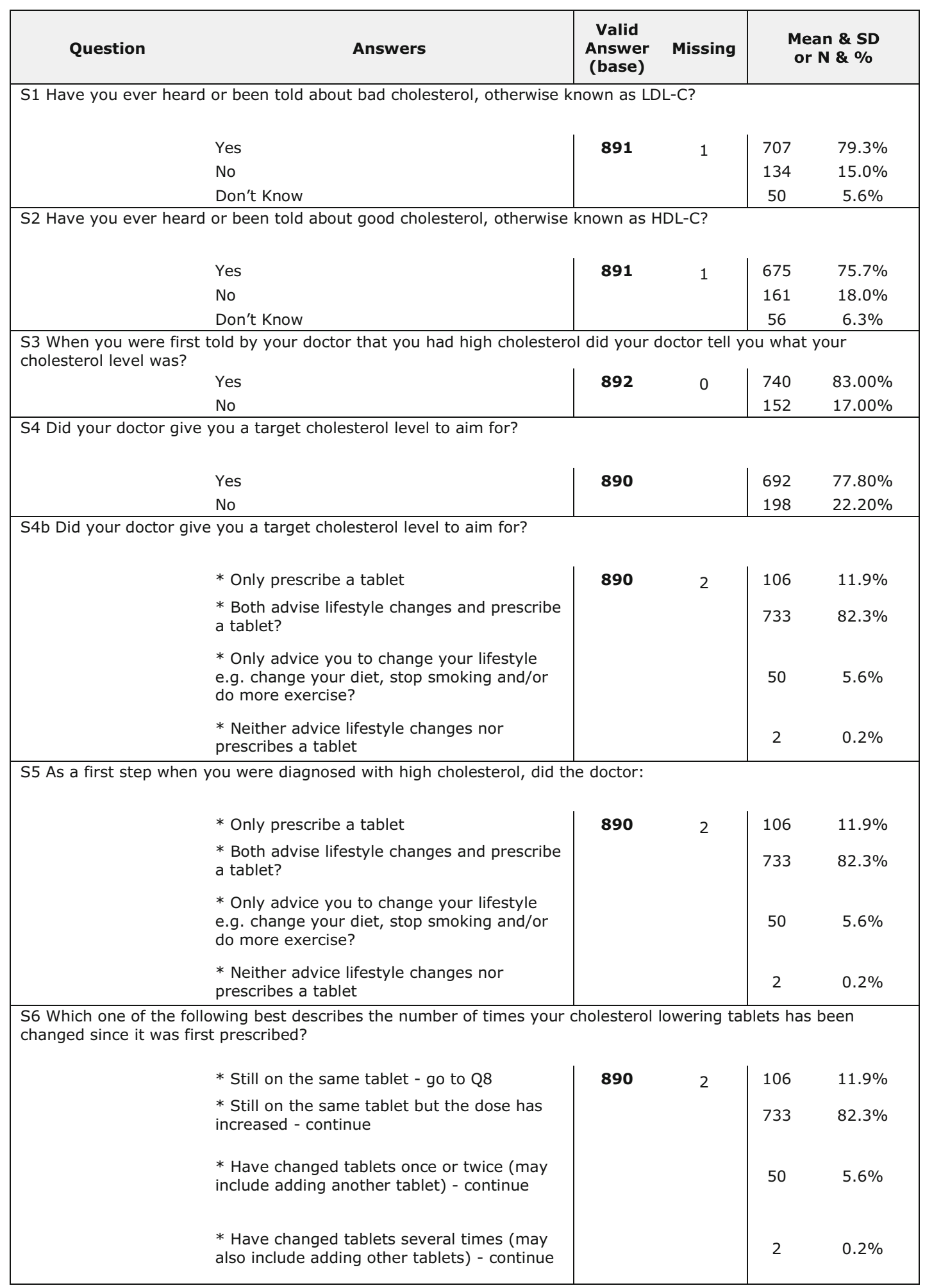


Table 5 continued

S7 How did you feel about your cholesterol lowering tablets having to be changed i.e. having the dose of your tablets increased or taking a different tablets?

\begin{tabular}{|c|c|c|c|c|}
\hline a. Satisfied & 892 & 0 & 249 & $27.9 \%$ \\
\hline $\begin{array}{l}\text { b. Concerned that your condition was now a } \\
\text { 'serious illness' }\end{array}$ & 892 & 0 & 130 & $14.6 \%$ \\
\hline c. No strong feelings & 892 & 0 & 81 & $9.1 \%$ \\
\hline d. Less motivated to keep taking your tablets & 892 & 0 & 32 & $3.6 \%$ \\
\hline $\begin{array}{l}\text { e. Irritated at having to keep making } \\
\text { changes }\end{array}$ & 892 & 0 & 79 & $8.9 \%$ \\
\hline $\begin{array}{l}\text { f. Disappointed that treatment was not } \\
\text { successful }\end{array}$ & 892 & 0 & 41 & $4.6 \%$ \\
\hline
\end{tabular}

S8 I am satisfied with the level of information available to me about high cholesterol:

\begin{tabular}{l|cc|cc} 
Agree & $\mathbf{8 9 2}$ & 0 & 700 & $78.50 \%$ \\
Disagree & & & 104 & $12 \%$ \\
Don't know/not applicable & & & 88 & $9.90 \%$
\end{tabular}

S9 I am frustrated that I still do not know whether my tablets have been effective enough in lowering my cholesterol.

\begin{tabular}{l|l|l|ll} 
Agree & $\mathbf{8 8 9}$ & 3 & 185 & $20.8 \%$ \\
Disagree & & & 602 & $68.0 \%$ \\
Don't know/not applicable & & & 102 & $11.5 \%$
\end{tabular}

S10 I always take my tablets to lower my cholesterol every day.

\begin{tabular}{|c|c|c|c|c|}
\hline Agree & 889 & 3 & 790 & $88.90 \%$ \\
\hline Disagree & & & 88 & $10 \%$ \\
\hline Don't know/not applicable & & & 11 & $1.20 \%$ \\
\hline
\end{tabular}

S11 I stopped taking my tablets when my cholesterol level returned to normal.

\begin{tabular}{l|c|cc} 
Agree & $\mathbf{8 8 9}$ & 155 & $17.4 \%$ \\
Disagree & & 690 & $78.0 \%$ \\
Don't know/not applicable & & 44 & $4.9 \%$ \\
\hline
\end{tabular}

S12 Sometimes I forgot to take my cholesterol lowering tablets.

\begin{tabular}{l|c|c|cc} 
Agree & $\mathbf{8 8 9}$ & 3 & 368 & $41.4 \%$ \\
Disagree & & & 505 & $57.0 \%$ \\
Don't know/not applicable & & & 16 & $1.8 \%$
\end{tabular}

S13 Approximately how often do you forget to take your cholesterol lowering tablets?
* Once a week
* Once every two weeks
* More than once a week
* Once a month or less

S14 How often do you think you can miss a tablet without affecting your cholesterol levels?
* Once a week
* Once every two weeks
* More than once a week
* Once a month or less

\begin{tabular}{l|ll}
871 & 215 & $24.7 \%$ \\
& 120 & $13.8 \%$ \\
& 156 & $17.9 \%$ \\
& 380 & $43.6 \%$
\end{tabular}


Table 5 continued

\begin{tabular}{|c|c|c|c|c|}
\hline \multicolumn{5}{|l|}{ S15 Which of the following best describes your current situation? } \\
\hline $\begin{array}{l}\text { * I have not reached my target cholesterol } \\
\text { level }\end{array}$ & \multirow[t]{4}{*}{887} & \multirow{4}{*}{5} & 151 & $17.0 \%$ \\
\hline $\begin{array}{l}\text { * I'm not sure whether I have reached my } \\
\text { target cholesterol level }\end{array}$ & & & 351 & $39.6 \%$ \\
\hline $\begin{array}{l}\text { * I have not been given a target cholesterol } \\
\text { level }\end{array}$ & & & 32 & $3.6 \%$ \\
\hline * I have reached my target cholesterol level & & & 353 & $39.8 \%$ \\
\hline \multicolumn{5}{|c|}{ S16 In general how do you feel about the way your high cholesterol has been treated? } \\
\hline a. Satisfied & 742 & 150 & 560 & $75.5 \%$ \\
\hline b. Motivated & 652 & 240 & 340 & $52.1 \%$ \\
\hline c. Concerned & 631 & 261 & 181 & $28.7 \%$ \\
\hline d. Frustrated & 589 & 303 & 40 & $6.8 \%$ \\
\hline e. Disappointed & 587 & 305 & 31 & $5.3 \%$ \\
\hline f. Confused & 591 & 301 & 68 & $11.5 \%$ \\
\hline g. No strong feelings & 609 & 283 & 176 & $28.9 \%$ \\
\hline \multicolumn{5}{|c|}{ S17 In general, how often do you see your doctor for a checkup of your cholesterol level? } \\
\hline Every 3 months & 883 & 9 & 377 & $42.7 \%$ \\
\hline Every 6 months & & & 246 & $27.9 \%$ \\
\hline More frequent than once every 3 months & & & 149 & $16.9 \%$ \\
\hline Every year & & & 64 & $7.2 \%$ \\
\hline Less often than once a year & & & 20 & $2.3 \%$ \\
\hline Do not have check-ups & & & 7 & $0.8 \%$ \\
\hline Don't know/ Can't remember & & & 20 & $2.3 \%$ \\
\hline
\end{tabular}

Finally, this study was an observational rather than interventional study, and as such, it suffers from many limitations. Most of the variables that may affect treatment goals were considered; however, many variables were not counted, such as differences in clinical practice between study investigators, lifestyle, diet factors, adverse events, and socioeconomic status.

\section{CONCLUSIONS}

Statins are the most frequently used lipid-lowering drugs and have a significant impact on achieving target LDL-C goals. As per the current CEPHEUS II results, hypercholesterolemia is still not being effectively managed in many at-risk patients in Egypt. The majority of patients enrolled in the study, all of whom were being actively treated with lipid-lowering medication, were considered at high risk of a cardiovascular event; hypercholesterolemia was particularly poorly managed in this group. Initiatives are needed to improve physicians' management of these patients with more focus on their risk profiles. Patient compliance to treatment is still urgently needed.

\section{ACKNOWLEDGEMENTS}

Sponsorship for this study and article processing charges were provided by an unrestricted research grant from AstraZeneca, Cairo, Egypt. All authors had full access to all of the data in this study and take complete responsibility for the integrity of the data and accuracy of the data analysis. Editorial assistance for the development of the manuscript was provided by 
Nagy Sobhy and Bola Megallaa of Nagy Research MEACRO, Cairo, Egypt, supported by AstraZeneca Egypt. All named authors meet the ICMJE criteria for authorship for this manuscript, take responsibility for the integrity of the work as a whole, and have given final approval for the version to be published.

Disclosures. Ashraf Reda, Alaa Etman, Ali Abdel-Rahim, Nabil Farag, Osama Sanad, and Sameh Salamah have nothing to disclose.

Compliance with Ethics Guidelines. All procedures followed were in accordance with the ethical standards of the responsible committee on human experimentation (institutional and national) and with the Helsinki Declaration of 1964, as revised in 2013. Informed consent was obtained from all patients for being included in the study. This article does not contain any new studies with human or animal subjects performed by any of the authors.

Open Access. This article is distributed under the terms of the Creative Commons Attribution-NonCommercial 4.0 International License (http://creativecommons.org/licenses/ by-nc/4.0/), which permits any noncommercial use, distribution, and reproduction in any medium, provided you give appropriate credit to the original author(s) and the source, provide a link to the Creative Commons license, and indicate if changes were made.

\section{REFERENCES}

1. The top 10 causes of death. World Health Organization. N.p., 2017. Web. 22 Feb. 2017.

2. Law MR, Wald NJ, Rudnicka AR. Quantifying effect of statins on low density lipoprotein cholesterol, ischaemic heart disease, and stroke: systematic review and meta-analysis. BMJ. 2003;326:1423.

3. EUROASPIRE Study Group. EUROSPIRE: a European Society of Cardiology survey of secondary prevention of coronary heart disease: principal results. Eur Heart J. 1997;18:1569-82.
4. De Backer G, Ambrosioni E, et al. European guidelines on cardiovascular disease prevention in clinical practice. Third joint task force of European and other societies on cardiovascular disease prevention in clinical practice. Eur Heart J. 2003;24:1601-10.

5. National Cholesterol Education Program (NCEP ATP III UPDATED 2004) Expert panel on detection, evaluation and, treatment of high blood cholesterol in adults (Adult Treatment Panel III). Third report of the National Cholesterol Education Program (NCEP ATP III UPDATED 2004) Expert panel on detection, evaluation and, treatment of high blood cholesterol in adults (Adult Treatment Panel III): final report. Circulation. 2002;106:3143-421.

6. Catapano AL, et al. ESC/EAS Guidelines for the management of dyslipidaemias. The task force for the management of dyslipidaemias of the European Society of Cardiology (ESC) and the European Atherosclerosis Society (EAS). Eur Heart J. 2016;37:2999-3058.

7. Elisaf MS, Nikas N. Centralized Pan-European survey on the undertreatment of hypercholesterolemia in patients using lipid-lowering drugs-the CEPHEUS-Greece survey. Angiology. 2010;61:465-74.

8. Reda A, Abdel-Rehim AA, Etman A, Afifi OSA. Centralized Pan-Middle East Survey on the under-treatment of hypercholesterolemia: results from the CEPHEUS Study in Egypt. Cardiol Therapy. 2014;3(1-2):27-40. doi:10.1007/s40119-0140031-x.

9. Hermans MP, Castro Cabezas M, Strandberg T, et al. Centralized Pan-European survey on the undertreatment of hypercholesterolaemia (CEPHEUS): overall findings from eight countries. Curr Med Res Opin. 2010;26:445-54.

10. Park JE, Chiang C-E, Munawar M, et al. Lipid-lowering treatment in hypercholesterolaemic patients: the CEPHEUS Pan-Asian survey. Eur J Prevent Cardiol. 2012;19(4):781-94.

11. Raal F, Schamroth C, Blom D, et al. CEPHEUS SA: a South African survey on the under-treatment of hypercholesterolaemia. Cardiovasc J Afr. 2011;22:234-40.

12. Chan RH, Chan PH, Chan KK, Lam SC, Hai JJ, Wong MK, Tam FC, Lam L, Chan CW, Lam YM, Siu DC, Tse HF, Lee SW. The CEPHEUS Pan-Asian survey: high low-density lipoprotein cholesterol goal attainment rate among hypercholesterolaemic patients undergoing lipid-lowering treatment in a Hong Kong regional centre. Hong Kong Med J. 2012;18(5):395-406. 
13. Wang Ko-Fan, Chang Chun-Chin, Wang Kang-Ling, et al. Determinants of low-density lipoprotein cholesterol goal attainment: insights from the CEPHEUS Pan-Asian Survey. 2014;77(2):61-7.

14. Munawar Muhammad, Hartono Beny, Rifqi Sodiqur. LDL cholesterol goal attainment in hypercholesterolemia: CEPHEUS Indonesian survey. Acta Cardiologica Sinica. 2013;29(1):71-81.
15. Sukonthasarn A, Homsanit M, Prommete B, Chotinaiwattarakul C, Piamsomboon C, Likittanasombat K. Lipid-lowering treatment in hypercholesterolemic patients: the CEPHEUS Thailand survey. J Med Assoc Thai. 2011;94(12):1424-34. 